\title{
The relationship between dietary patterns, dietary quality index, and dietary inflammatory index with the risk of all types of cancer: Golestan cohort study
}

\author{
Marzieh Nojomi ${ }^{1,2}$, Arash Tehrani Banihashemi ${ }^{3}$, Hassan Niksima $^{3}$, Maryam Hashemian $^{4}$, Azadeh Mottaghi ${ }^{5 *}$ (D), \\ Reza Malekzaddeh 6
}

Received: 1 Jul 2019

Published: 13 Apr 2021

\section{Abstract}

Background: Dietary patterns and diet quality index (DQI) are widely discussed in relation with different health conditions and have recently been taken into consideration for all cancer types. Since chronic inflammation has been recognized as an important biologic risk factor for cancer occurrence, especially in epithelial tissues, proinflammatory or anti-inflammatory characteristics of diet has become the center of attention. In the present study, we aimed to identify whether a specific dietary pattern, Mediterranean dietary score (MDs), and dietary inflammatory index (DII) were associated with overall cancer risk in Iranian population.

Methods: This study was performed in the context of the Golestan cohort study. Participants with extreme daily energy intake or those who did not answer more than 30 question of the Food Frequency questionnaire (FFQ) were excluded. Dietary patterns, MDs, and DII were measured from FFQ. Age, sex, total energy, place of residence, smoking, wealth score, ethnicity, opiate use, BMI, education, marital status, and physical activity score were considered as confounding variables. Using Cox proportional hazards regression models, hazard ratios (HRs) and 95\% confidence interval of cancer were estimated.

Results: HRs (95\% CIs) of all cancers by quartiles of Western dietary pattern, DII, and MDs showed that the forth quartile of the Western dietary pattern is attributed to $23 \%$ higher cancer risk (HRs: 1.23 , CI: $1.09-1.40, \mathrm{P}<0.001$, adjusted for age and sex) compared to the first quartile. It also remained significant after further adjustments (HRs $=1.20, \mathrm{CI}: 1.06-1.36, \mathrm{P}<0.001)$. There was a higher cancer risk in the fourth quartile of DII in comparison with the first quartile (HRs $=1.16$, CI: 1.01-1.32, P trend $<0.001$, adjusted for age and sex). The lower adherence to the Mediterranean dietary pattern also largely contributes to $27 \%$ higher cancer risk (HRs: 1.27, CI: 1.12-1.44), P trend $<0.001$, adjusted for age and sex), which also remained remarkable after further adjustments ((HRs $=1.19, \mathrm{CI}: 1.05-1.35, \mathrm{P}$ trend $<0.001)$

Conclusion: Cancer is highly correlated to dietary intake and dietary patterns, such as the Western dietary pattern, while the Mediterranean diet score was inversely associated with cancer risk. Further investigations are required to get a broader insight into cancer determinants in population.

Keywords: Dietary Patterns, Dietary Quality Index, Dietary Inflammatory Index, Risk, Cancer, Golestan Cohort Study

Conflicts of Interest: None declared

Funding: Non- communicable Diseases Committee (No. 942256) of the National Institute for Medical Research Development (NIMAD), Tehran, Iran

*This work has been published under CC BY-NC-SA 1.0 license.

Copyright $\odot$ Iran University of Medical Sciences

Cite this article as: Nojomi M, Tehrani Banihashemi A, Niksima H, Hashemian M, Mottaghi A, Malekzaddeh R. The relationship between dietary patterns, dietary quality index, and dietary inflammatory index with the risk of all types of cancer: Golestan cohort study. Med J Islam Repub Iran. 2021 (13 Apr);35:48. https://doi.org/10.47176/mjiri.35.48

Corresponding author: Dr Azadeh Mottaghi, mottaghi.a@iums.ac.ir

1. Preventive Medicine \& Public Health Research Center, Psychosocial Health Research Institute, Department of Community and Family Medicine, School of Medicine, Iran University of Medical Sciences, Tehran, Iran

2. Department of Sociology \& Anthropology, Nipissing University, North Bay, Ontario, Canada

3. Preventive Medicine \& Public Health Research Center, Department of Community and Family Medicine, School of Medicine, Iran University of Medical Sciences, Tehran, Iran

4. Metabolic Epidemiology Branch, Division of Cancer Epidemiology and Genetics, National Cancer Institute, Bethesda, Maryland, USA

5. Research Center for Prevention of Cardiovascular Disease, Institute of Endocrinology \& Metabolism, Iran University of Medical Sciences, Tehran, Iran

6. Digestive Diseases Research Center, Digestive Diseases Research Institute, Tehran University of Medical Sciences, Tehran, Iran $\uparrow$ What is “already known" in this topic:

There is a large body of evidence on a correlation between dietary intakes and all types of cancer.

\section{$\rightarrow$ What this article adds:}

The association between dietary patterns, diet quality index, and dietary inflammatory index with cancer incidence was assessed in the present article. The Western dietary pattern, inflammatory diet, and lower adherence to the Mediterranean dietary pattern contribute to $23 \%, 16 \%$, and $27 \%$ higher cancer risk, respectively. 


\section{Introduction}

Cancer is one of the most important causes of death worldwide, accounting for $21 \%$ of the overall mortality (1), as it is estimated that its incidence will increase by $70 \%$ in the next 20 years (2). The number of new cancer cases reached 14.1 million in 2012 and is expected to rise to 23.6 million new cases in 2030 (3). Cancer etiology has been widely the center of attention to find major risk factors and consequently discover therapeutic modalities and cancer preventing intervention $(4,5)$. Since about one third of cancer deaths are associated with major behavioral and dietary risk factors like unhealthy diet or low fresh fruit and vegetable consumption, it is of great importance to further offset the effects of such risk factors (5).

Dietary patterns and diet quality index (DQI) are widely discussed in relation with different health conditions and have also been recently taken into consideration for all cancer types $(6,7)$. Dietary patterns assess the effects of whole diet, rather than individual nutrients or foods, enabling investigators to examine associations with overall diet (8). In the Dietary Patterns Methods Project, 4 indexes of diet (the Alternative Healthy Eating Index 2010 (AHEI-2010), the alternate Mediterranean diet score (aMED), and the Dietary Approaches to Stop Hypertension (DASH) were assessed in relation to colorectal cancer. Results of the mentioned study showed that all 4 diet quality indexes were inversely associated with colorectal cancer risk in both men and women, although the associations for the AHEI-2010 and aMED score after adjustment for potential confounding factors did not remain statistically significant in women (9).

A low-fat dietary pattern is reported to be associated with reduced pancreatic cancer incidence in women who were overweight or obese and fewer ovarian cancers among postmenopausal women $(10,11)$. Likewise, a specific dietary pattern that correlated with C-peptide concentrations was associated with an increase in colon cancer, especially among women who were overweight or sedentary (12). However, another survey found no association between a vegetarian pattern or a salty pattern and breast cancer (13). The Western dietary pattern also increased prostate cancer risk (14). Diet quality index is also composed of a combination of foods and/or nutrient components reflecting dietary guidelines and complexity of diet $(15,16)$. It seems that the Mediterranean dietary pattern as one of the dietary quality indices can reduce breast cancer risk as well as overall cancer risk $(17,18)$. Chronic inflammation has been also recognized as an important biologic risk factor for cancer occurrence, especially in epithelial tissues (19). As diet causes exposures to many different agents that can have proinflammatory or antiinflammatory characteristics, its role in chronic inflammation and cancer development has been recently widely discussed, although data about the inflammatory potential of common diet in Iran is scarce or nonexistent. In 2014, an inflammatory index was developed to assess the inflammatory potential of an individual's diet (20). Some studies have shown an association between inflammatory potential of an individual's diet and incidence of different cancers like colorectal cancers (21-24), renal carcinoma
(25), esophageal squamous cell carcinoma $(26,27)$, hepatocellular (28), lung (29), prostate (30-32), and breast cancer $(33,34)$.

In the present study, we aimed to identify whether a specific dietary patterns, MDs and dietary inflammatory index, were associated with overall cancer risk in an Iranian population.

\section{Methods}

\section{Study population}

This study is a part of the Golestan cohort study done in Golestan province located in the northeast of Iran. Golestan cohort study recruited about 50000 healthy persons aged between 40 and 75 years during 2004 and 2008 to investigate the etiology of esophageal cancer; all details are published elsewhere (35). Some participants were excluded from the study because of extreme daily energy intake, defined as $>99$ th percentile or less than the first percentile, or with missed $>30$ responses on the FFQ. The ethical review committee of the Digestive Diseases Research Institute approved the study protocol.

\section{Data collection and measures}

Characteristics of the participants, including age, smoking status, ethnicity, education levels, history of diseases physical activity levels, and family history of cancer, were obtained through face-to-face interviews using a general questionnaire at the beginning of the study. Weight was measured using digital scales, while participants were minimally clothed, without shoes to the nearest $100 \mathrm{~g}$. Height was measured using a nonstretchable tape meter in a standing position without shoes to the nearest $0.5 \mathrm{~cm}$. The body mass index (BMI) was calculated as weight $(\mathrm{kg})$ divided by square of the height $\left(\mathrm{m}^{2}\right)$. Since physical activity at work is considered to be mostly staple, it was used to calculate a physical activity score (36). Using household appliances, vehicles, and other variables associated with wealth through multiple correspondence analysis wealth score was estimated (37). Marital status was considered as single or married. Because the number of divorced or widowed individuals was small, they were categorized in a single category.

\section{Dietary intake}

The dietary intake was measured using a validated 116item Food Frequency Questionnaire (FFQ), which was designed especially for GCS population (38). Participants were asked about their intake frequency for each food item consumed during the past year on a daily, weekly, or monthly basis; portion sizes of consumed foods reported in household measures were then converted to grams. To analyze food items in terms of the energy and nutrient intake of participants, the Iranian Food Composition Table and the food composition tables (FCT) of the United States Department of Agriculture (USDA) were used.

\section{Dietary pattern}

In order to determine dietary patterns, the principle 
component analysis (PCA) was applied based on dietary information collected from the 18 food groups (Table 1) based on the similarity in their nutrient contents. Data of food groups were standardized and then considering $\pm 4 \mathrm{SD}$, data were truncated. Skewed data were normalized using logarithm method, and then data were energy adjusted by the residual methods. The factors were rotated with varimax rotation. Considering Eigen values $>1$, the scree plot, and the interpretability of the factors, 3 patterns were identified. Items which have absolute correlation $\geq 0.2$ with that factor were considered to load on a factor and retained in the calculation of the dietary pattern score (39). The Kaiser-Mayer-Olkin statistic was 0.88, which indicate a good appropriateness of factor analysis. This statistic considers a measure of sampling adequacy. To evaluate the suitability of the correlation matrix for factor analysis, Bartlett's test of sphericity was used; $P$ value for Bartlett's test of sphericity was $<0.001$. Factor scores of the participants were calculated using sum of multiplying the intake of the standardized food groups by their respective factor loadings on each pattern. The factor loading of the 3 dietary patterns extracted by factor analysis is presented in Table 2.

Among the 3 extracted dietary patterns, only the Western dietary pattern, which correlated with cancer risk, was reported.

\section{Mediterranean diet score (MDs)}

The Mediterranean diet score was computed according to data obtained from FFQ. Seven components (saturated fatty acids, cholesterol, meat, olive oil, fish, cereals, and vegetables + fruits) were assessed in this index and score of 0,1 , or 2 were assigned to the daily intake of each of the mentioned component. A total score ranged between 0 and 14, with lower scores indicating a high quality of diet and better adherence to the Mediterranean dietary pattern (40).

\section{Dietary inflammatory index}

To calculate the dietary inflammatory index, we used 31 food items that were available in our food composition database and we also applied the standardized method

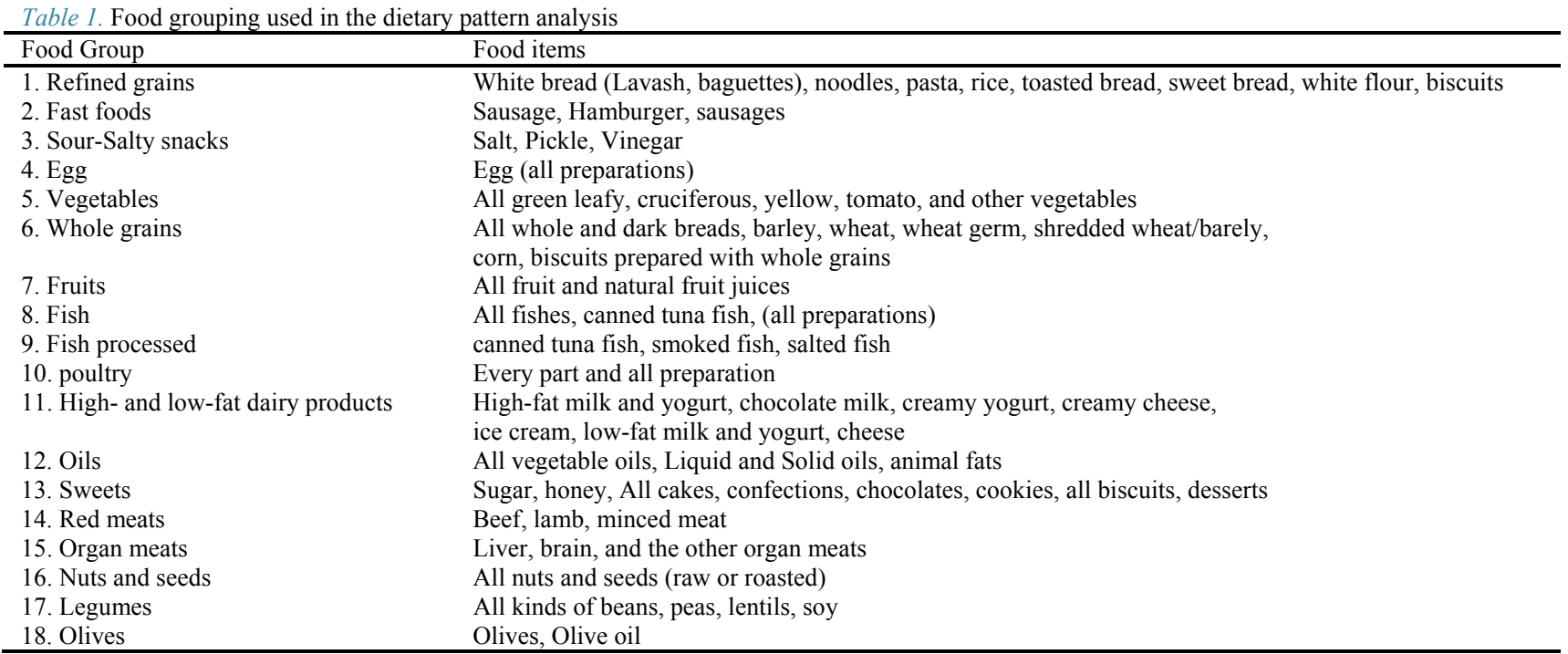

\begin{tabular}{lcc} 
& & \\
Table 2. Factor loading of 3 dietary patterns extracted by factor analysis & & \\
\hline Food Groups & Healthy dietary pattern & Western dietary pattern \\
\hline Fruit and dried fruit & $0.662^{\mathrm{a}}$ & \\
Refined grains & -0.597 & \\
Olive oil & 0.551 & \\
High- and low-fat dairy products & 0.452 & \\
Poultry and fish & 0.327 & \\
Liquid oils except olive oil & 0.240 & 0.677 \\
Canned products & 0.279 & 0.603 \\
Carbonated drinks & & 0.573 \\
Fast foods & & 0.581 \\
Salty snacks & & 0.311 \\
Mayonnaise & & \\
Organ meats & & \\
Legumes & & \\
potatoes & & 0.635 \\
Egg & & 0.639 \\
Red meats & & \\
Tea & & 0.477 \\
Percentage of variance explained & & \\
\hline
\end{tabular}

${ }^{a}$ Values are factor loading of dietary patterns $(n=2141)$. Factor loading $\leq 0.2$ are not shown. ${ }^{b}$ Eigen value $>1$. 
provided by Shivappa (9). These food items were vitamin B12, vitamin B6, $\beta$-carotene, caffeine, carbohydrate, cholesterol, energy, fat, fiber, folic acid, iron, magnesium, niacin, protein, riboflavin, selenium, thiamin, vitamin A, vitamin $C$, vitamin $D$, zinc, trans fatty acid, saturated fat acids (SFA), monounsaturated fat acids (MUFA), polyunsaturated fatty acids (PUFA), garlic, onion, saffron, turmeric, black tea, and pepper. The calculation method has been described elsewhere in detail (9). Briefly, a standard mean for each food item from the representative world database was subtracted from the daily intake and divided by its standard deviation to generate $\mathrm{Z}$ scores. For minimizing the effect of right skewing, the percentile value was converted to percentile score using SPSS and these values were divided by 100 to calculate the scoring of $0-1$. Also, to generate a centered percentile score on 0 (null) and bounded between -1 (maximally anti-inflammatory) and +1 (maximally pro-inflammatory), each percentile score is doubled and then ' 1 ' is subtracted. Then, these values were multiplied by the respective overall food parameter-specific inflammatory effect score to obtain the 'food parameter specific DII score'. Finally, all of the food parameter-specific DII scores are summed to generate the overall DII score for an individual.

\section{Statistical analysis}

Using Cox proportional hazards regression models, HRs and $95 \%$ CIs were estimated. Using Aalen plots and the Schoenfeld residuals test, the proportional hazards assumption was checked. Age was used as the underlying time metric. All possible confounders were investigated; namely, age, sex, total energy, place of residence, smoking, wealth score, ethnicity, opiate use, BMI, education, marital status, and physical activity score. The model was not adjusted for alcohol drinking, as only few participants reported drinking alcohol, and it is not deemed to be a risk factor in the corresponding area (41).

Multivariate HRs were reported within quartiles where the lowest quartile was considered as the reference category. For linear trend tests, the median value of each quartile was used. We also conducted stratified analyses by age, sex, BMI, ethnicity, and residence area. Statistical analyses were performed by using STATA software (version 12; StataCorp). Reported P values are 2-sided.

\section{Results}

Among the participants, 49940 were included in the final analysis (21 207 men and 28733 women). During a median follow-up time of 10.1 years (IQR: 9.16-11.18 y), 1932 cases were diagnosed with any type of cancer. Baseline characteristics are shown according to the quartile of the dietary pattern, Med-DQI, and DII separately (Tables 3, 4, and 5). A higher adherence to the Western dietary pattern was associated with increased energy intake and smoking, and lower wealth score in the fifth category and also lower education level. Towards higher quartiles of MDs representing lower adherence to the Mediterranean dietary pattern, lower energy intake and BMI, and decreased wealth score in most categories were indicated. Upper quartiles of DII were also associated with lower BMI and energy intake and also lower wealth score in higher categories.

Table 6 indicates HRs (95\% CIs) of all cancers, by quartiles of the Western dietary pattern, DII, and MDs. The fourth quartile of the Western dietary pattern is attributed to $23 \%$ higher cancer risk (HRs: 1.23 , CI: 1.09$1.40, \mathrm{P}<0.001$, adjusted for age and sex) compared to the first quartile. It also remained significant after further adjustments $(\mathrm{HRs}=1.20, \mathrm{CI}: 1.06-1.36, \mathrm{P}<0.001)$. There was a higher cancer risk in the fourth quartile of DII in comparison with the first quartile (HRs $=1.16$, CI: 1.01 1.32, $\mathrm{P}$ trend $<0.001$, adjusted for age and sex). Lower

Table 3. Baseline characteristics of participants by quartiles of the Western dietary pattern ${ }^{1}$

\begin{tabular}{|c|c|c|c|c|}
\hline $\begin{array}{l}\text { Quartile } \\
\text { Variable }\end{array}$ & Q1 & Q2 & Q3 & Q4 \\
\hline Western Dietary Pattern score & $-0.99 \pm 1.36$ & $0.14 \pm 0.15$ & $1.13 \pm 0.46$ & $6.46 \pm 10.54$ \\
\hline Male sex, $\mathrm{n}(\%)$ & $5179(41.7)$ & $5185(43.1)$ & $5327(43.5)$ & $5114(41.7)$ \\
\hline Age (y) & $52.38 \pm 9.15$ & $51.90 \pm 8.81$ & $51.91 \pm 8.91$ & $52.24 \pm 9.16$ \\
\hline Energy (kcal) & $2124 \pm 590$ & $2241 \pm 601$ & $2240 \pm 627$ & $2141 \pm 1826$ \\
\hline BMI $\left(\mathrm{kg} / \mathrm{m}^{2}\right)$ & $26.60 \pm 5.45$ & $26.82 \pm 5.40$ & $26.78 \pm 5.43$ & $26.51 \pm 5.49$ \\
\hline Smoker, n (\%) & $2731(22)$ & $2489(20.7)$ & $2647(21.6)$ & $2802(22.8)$ \\
\hline \multicolumn{5}{|l|}{ Wealth score ${ }^{3}, \mathrm{n}(\%)$} \\
\hline 1 & $2620(21.10)$ & $2345(19.5)$ & $2339(19.1)$ & $3007(24.5)$ \\
\hline 2 & $2283(18.4)$ & $2238(18.6)$ & $2229(18.2)$ & $2296(18.7)$ \\
\hline 3 & $2618(21.1)$ & $2774(23.1)$ & $2738(22.3)$ & $2559(20.9)$ \\
\hline 4 & $2295(18.5)$ & $2434(20.2)$ & $2446(20)$ & $2176(17.7)$ \\
\hline 5 & $2599(20.9)$ & $2231(18.6)$ & $2503(20.4)$ & $2232(18.2)$ \\
\hline \multicolumn{5}{|l|}{ Physical activity score, $\mathrm{n}(\%)$} \\
\hline Low & $4414(35.6)$ & $4118(34.3)$ & $4346(35.5)$ & $4448(36.3)$ \\
\hline Intermediate & $4086(32.9)$ & $3707(30.8)$ & $3809(31.1)$ & $3848(31.4)$ \\
\hline High & $3892(31.3)$ & $4176(34.7)$ & $4069(33.2)$ & $3937(32.1)$ \\
\hline Rural place of residence, $\mathrm{n}(\%)$ & $10335(83.2)$ & $9966(82.9)$ & $10075(82.2)$ & $10080(82.2)$ \\
\hline Turkmen ethnicity, n (\%) & $8157(65.7)$ & $9739(81)$ & $9393(76.6)$ & $9026(73.6)$ \\
\hline Opium user, n (\%) & $2033(16.4)$ & $2010(16.7)$ & $2057(16.8)$ & $2222(18.1)$ \\
\hline Marital status, single, n (\%) & $1582(12.7)$ & $1307(10.9)$ & $1382(11.3)$ & $1684(13.7)$ \\
\hline No formal education, $\mathrm{n}(\%)$ & $8782(70.7)$ & $8520(70.9)$ & $8435(68.8)$ & $8561(69.8)$ \\
\hline
\end{tabular}




\begin{tabular}{|c|c|c|c|c|}
\hline $\begin{array}{l}\text { Quartile } \\
\text { Variable }\end{array}$ & Q1 & Q2 & Q3 & $\overline{\mathrm{Q} 4}$ \\
\hline MD score & $2.48 \pm 0.44$ & $3.35 \pm 0.18$ & $4.03 \pm 0.23$ & $6.11 \pm 2.09$ \\
\hline Male sex, n (\%) & $7399(60.5)$ & $5846(47.6)$ & $4586(37.4)$ & $2975(24.4)$ \\
\hline Age $(y)$ & $51.63 \pm 8.71$ & $51.41 \pm 8.75$ & $51.78 \pm 8.85$ & $53.62 \pm 9.55$ \\
\hline Energy (kcal) & $2922 \pm 1781$ & $2335 \pm 265.58$ & $1998 \pm 211.23$ & $1489 \pm 301.73$ \\
\hline BMI $\left(\mathrm{kg} / \mathrm{m}^{2}\right)$ & $27.12 \pm 5.27$ & $26.83 \pm 5.33$ & $26.67 \pm 5.41$ & $26.10 \pm 5.72$ \\
\hline Smoker, n (\%) & $3428(28)$ & $2872(23.4)$ & $2336(19.1)$ & $2034(16.7)$ \\
\hline \multicolumn{5}{|l|}{ Wealth score ${ }^{3}, \mathrm{n}(\%)$} \\
\hline 1 & $2181(17.8)$ & $2404(19.6)$ & $2563(20.9)$ & $3164(25.9)$ \\
\hline 2 & $1912(15.6)$ & $2245(18.3)$ & $2322(19)$ & $2569(21)$ \\
\hline 3 & $2363(19.3)$ & $2767(22.5)$ & $2872(23.4)$ & $2689(22)$ \\
\hline 4 & $2423(19.8)$ & $2512(20.5)$ & $2353(19.2)$ & 2064 (16.9) \\
\hline 5 & $3344(27.4)$ & $2353(19.2)$ & $2142(17.5)$ & $1726(14.1)$ \\
\hline \multicolumn{5}{|l|}{ Physical activity score, n (\%) } \\
\hline Low & $4954(40.5)$ & $4275(32.4)$ & $3965(33.1)$ & 4135 (33.9) \\
\hline Intermediate & $3489(28.5)$ & $3977(32.5)$ & $7399(31.5)$ & $4205(34.4)$ \\
\hline High & $3750(30.7)$ & $4285(35)$ & $8267(35.2)$ & $3842(31.5)$ \\
\hline Rural place of residence, $\mathrm{n}(\%)$ & $9319(76.2)$ & $9931(80.9)$ & $10412(85)$ & $10799(88.4)$ \\
\hline Turkmen ethnicity, n (\%) & $9243(75.6)$ & $9332(76)$ & $9193(75)$ & $8550(70)$ \\
\hline Opium user, n (\%) & $2247(18.4)$ & $2053(16.7)$ & $1881(15.4)$ & $2141(17.5)$ \\
\hline Marital status, single, $\mathrm{n}(\%)$ & $976(8)$ & $1155(9.4)$ & $1505(12.3)$ & $2319(19)$ \\
\hline No formal education, $\mathrm{n}(\%)$ & $7050(57.7)$ & $8257(67.2)$ & $8997(73.4)$ & $10000(81.9)$ \\
\hline
\end{tabular}

${ }^{1} \mathrm{n}=49940$

${ }^{2}$ Mean \pm SD (all such values)

${ }^{3}$ Wealth score was estimated through multiple correspondence analysis on the basis of household appliances, vehicles, and other variables associated with wealth

Table 5. Baseline characteristics of participants in the Golestan cohort study by quartiles of dietary inflammatory index (DII) ${ }^{1}$

\begin{tabular}{|c|c|c|c|c|}
\hline $\begin{array}{l}\text { Quartile } \\
\text { Variable }\end{array}$ & Q1 & Q2 & Q3 & Q4 \\
\hline DII score & $-1.05 \pm 0.32$ & $-0.32 \pm 0.18$ & $0.39 \pm 0.24$ & $1.90 \pm 1.20$ \\
\hline Male sex, n (\%) & $6785(55.5)$ & $5999(48.9)$ & $4783(39.2)$ & $3248(26.4)$ \\
\hline Age (y) & $51.32 \pm 8.61$ & $51.51 \pm 8.74$ & $52.07 \pm 9$ & $53.53 \pm 9.52$ \\
\hline Energy (kcal) & $2621 \pm 560$ & $2488 \pm 1688$ & $2072 \pm 691$ & $1565 \pm 360$ \\
\hline $\mathrm{BMI}\left(\mathrm{kg} / \mathrm{m}^{2}\right)$ & $27.67 \pm 5.26$ & $26.80 \pm 5.35$ & $26.47 \pm 5.41$ & $25.80 \pm 5.60$ \\
\hline Smoker, n (\%) & $3206(26.2)$ & $2910(23.7)$ & $2546(20.9)$ & $2013(16.4)$ \\
\hline \multicolumn{5}{|l|}{ Wealth score ${ }^{3}, \mathrm{n}(\%)$} \\
\hline 1 & $1375(11.2)$ & 2435 (19.9) & $2765(22.7)$ & $3742(30.4)$ \\
\hline 2 & $1541(12.6)$ & $2226(18.2)$ & $2460(20.2)$ & $2827(23)$ \\
\hline 3 & $2218(18.1)$ & $2777(22.6)$ & $2895(23.7)$ & $2802(22.8)$ \\
\hline 4 & $2752(22.5)$ & $2472(20.2)$ & $2274(18.6)$ & $1860(15.1)$ \\
\hline 5 & $4346(35.5)$ & $2353(19.2)$ & $1802(14.8)$ & $1066(8.7)$ \\
\hline \multicolumn{5}{|l|}{ Physical activity score, n (\%) } \\
\hline Low & $4770(39)$ & $4297(35)$ & $3978(32.6)$ & $4293(34.9)$ \\
\hline Intermediate & $3631(29.7)$ & $3756(30.6)$ & $3938(32.3)$ & $4131(33.6)$ \\
\hline High & $3798(31)$ & $4180(34.1)$ & $4260(34.9)$ & $3844(31.3)$ \\
\hline Rural place of residence, $n(\%)$ & $9462(77.4)$ & $9893(80.7)$ & $10239(84)$ & $10884(88.5)$ \\
\hline Turkmen ethnicity, n (\%) & $7391(60.4)$ & $9423(76.8)$ & $9752(80)$ & $9772(79.5)$ \\
\hline Opium user, n (\%) & $2007(16.4)$ & $2072(16.9)$ & $2104(17.3)$ & $2146(17.5)$ \\
\hline Marital status, single, n (\%) & $1053(8.6)$ & $1175(9.6)$ & $1500(12.3)$ & $2230(18.1)$ \\
\hline No formal education, $\mathrm{n}(\%)$ & $6123(50.1)$ & $8317(67.8)$ & $9246(75.8)$ & $10632(86.5)$ \\
\hline
\end{tabular}

${ }^{1} \mathrm{n}=49940$

${ }^{2}$ Mean \pm SD (all such values)

${ }^{3}$ Wealth score was estimated through multiple correspondence analysis on the basis of household appliances, vehicles, and other variables associated with wealth

adherence to the Mediterranean dietary pattern also largely contributes to $27 \%$ higher cancer risk (HRs: $1.27, \mathrm{CI}$ : $1.12-1.44)$, $\mathrm{P}$ trend $<0.001$, adjusted for age and sex), it also remained remarkable after further adjustments (HRs $=1.19, \mathrm{CI}: 1.05-1.35, \mathrm{P}$ trend $<0.001)$.

\section{Discussion}

In the present population-based survey, cancer incidence was associated with higher adherence to the Western dietary pattern, higher DII, and lower adherence to the Mediterranean diet. Apart from the results referring to DII, all findings remained significant after adjustment for all confounding variables.

Among the 3 dietary patterns extracted from the dietary intakes of the participants, the Western dietary pattern was associated with the risk of cancer. This dietary pattern was rich in carbonated drinks, fast foods, salty snacks, and mayonnaise. It was reported to increase cancer risk by $20 \%$ in this study. Red meat and tea were classified under mixed dietary pattern. Different methods of red meat processing such as grilling can be effective in making carcinogens such as heterocyclic amines. On the other hand, hot tea consumption can also contribute to esophageal cancer.

Diet is widely discussed as a modifiable determinant for cancer, as healthy dietary patterns seem to be related to decreased risk of colon and breast cancer and unhealthy dietary patterns can lead to increased risk of colon cancer $(42,43)$. However, it can no longer be generalized about 
Table 6. Hazard ratios (95\% CIs) of all cancer by quartiles of the Western dietary pattern, DII, and MDS score

\begin{tabular}{|c|c|c|c|c|c|}
\hline $\begin{array}{l}\text { Quartile } \\
\text { Variable }\end{array}$ & Q1 & Q2 & Q3 & Q4 & P-trend \\
\hline \multicolumn{6}{|c|}{ Western Dietary pattern } \\
\hline Score & $-0.55(0.91)^{1}$ & $0.11(0.25)$ & $1.06(0.78)$ & $4.26(3.94)$ & \\
\hline Person-years & 123768 & 120700 & 122731 & 122673 & \\
\hline Cases (n) & 448 & 426 & 482 & 532 & \\
\hline $\operatorname{HR}(95 \% \mathrm{CI})^{2}$ & 1 & $1.01(0.88-1.15)$ & $1.12(0.99-1.28)$ & $1.23(1.09-1.40)$ & $<0.001$ \\
\hline $\operatorname{HR}(95 \% \mathrm{CI})^{3}$ & 1 & $0.99(0.86-1.13)$ & $1.11(0.98-1.26)$ & $1.20(1.06-1.36)$ & $<0.001$ \\
\hline \multicolumn{6}{|l|}{ DII } \\
\hline Score & $-0.98(0.44)$ & $-0.33(0.32)$ & $0.37(0.42)$ & $1.58(1.11)$ & \\
\hline Person-years & 125750 & 123574 & 121412 & 119425 & \\
\hline Cases (n) & 444 & 435 & 484 & 527 & \\
\hline $\operatorname{HR}(95 \% \mathrm{CI})^{2}$ & 1 & $0.98(0.86-1.12)$ & $1.08(0.95-1.23)$ & $1.16(1.01-1.32)$ & $<0.001$ \\
\hline $\operatorname{HR}(95 \% \mathrm{CI})^{3}$ & 1 & $0.92(0.80-1.05)$ & $0.99(0.87-1.14)$ & $1.03(0.89-1.19)$ & $<0.001$ \\
\hline \multicolumn{6}{|l|}{ MDS } \\
\hline Score & $2.59(0.58)$ & $3.36(0.31)$ & $4.01(0.40)$ & $5.49(1.73)$ & \\
\hline Person-years & 125632 & 123662 & 234095 & 117300 & \\
\hline Cases (n) & 468 & 463 & 878 & 524 & \\
\hline $\operatorname{HR}(95 \% \mathrm{CI})^{2}$ & 1 & $1.04(0.91-1.18)$ & $1.11(1.00-1.24)$ & $1.27(1.12-1.44)$ & $<0.001$ \\
\hline $\operatorname{HR}(95 \% \mathrm{CI})^{3}$ & 1 & $1.02(0.90-1.17)$ & $1.07(0.95-1.19)$ & $1.19(1.05-1.35)$ & $<0.001$ \\
\hline
\end{tabular}

HR $(95 \% \mathrm{CI})$

${ }^{2}$ Adjusted for age (y) and sex

${ }^{3}$ Adjusted for age (y), sex, total energy (kcal/d), place of residence (urban or rural), smoking (never or ever), wealth score (1-5), ethnicity (non-Turkmen or Turkmen),

opiate use (never or ever), BMI $\left(\mathrm{kg} / \mathrm{m}^{2}\right)$, education (illiterate or formal education), marital status (single or married), and physical activity score (categorical)

different cancer types and varied target populations, since these results are inconsistent with some of previous studies (44-46). There may not be correlations between unhealthy dietary pattern and risk of upper digestive tract, pancreatic, ovarian, endometrial, and prostatic cancers based on case-control studies $(44,47)$. On the other hand, it is also likely that dietary parameters can directly or indirectly affect cancer incidence. A balanced ratio of fatty acids, the high fiber content, and the substantial amounts of antioxidant compounds result in suppressing multiple cancer-related biological pathways, including carcinogen bio activation, cell signaling, cell cycle regulation, angiogenesis, and inflammation (2). Altered energy imbalance, excess body weight, adverse homeostasis alterations are also the indirect consequences of improper eating habits. Moreover, unhealthy dietary patterns mostly contain salt, $\mathrm{N}$-nitros compounds, heterocyclic amines, heme iron, and polycyclic aromatic hydrocarbons, which are deemed to be responsible for the carcinogenic effects (48). Accordingly, a low-fat dietary pattern is proposed to reduce the incidence of ovarian cancer among postmenopausal women and pancreatic cancer incidence in women who were overweight or obese $(10,11)$.

In the model by which we calculated MDs, a higher score is associated with lower adherence to the Mediterranean diet; thus, across the upper quartiles of MDs, $19 \%$ increase in cancer risk is reported. In other word, the more participants were adhered to the Mediterranean diet, the less cancer risk they were exposed to. Adherence to MDs is considered as a remarkable protective factor for cancer incidence, decreasing the risk of almost all cancers (18).

The Mediterranean dietary pattern has a protective role for breast, female genital tract, urinary tract, and a few other epithelial neoplasms (49). Various antioxidants and other micronutrients provided by the Mediterranean diet has an inverse relationship with cancer risk, but the main components responsible for the favorable effect of a diet rich in vegetables and fruit remain undefined. Fish is an- other useful component of this dietary pattern (50). On the contrary, red meat consumption is related to several common neoplasms. Another component of the Mediterranean diet that has a preventive role in cancer is whole-grain foods that are related to a reduced risk of several types of cancer, particularly of the upper digestive tract. This may be due to an effective role of fiber. However, refined grain intake and, consequently, glycaemic load and glycaemic index were associated with increased risk of different types of cancer, including breast and colorectal, among others (51).

There was also a suggestive positive association between a higher DII score and cancer risk among the participants. Diet seems to be closely related to chronic inflammation status $(52,53)$. Some studies have shown an association between inflammatory potential of an individual's diet and incidence of different cancers like colorectal cancers (16-19), renal carcinoma (20), esophageal squamous cell carcinoma $(21,22)$, hepatocellular (23), lung (24), prostate (25-27), and breast cancer $(28,29)$. Oxidative stress and reduced antioxidant defense system are also associated with cell injury and aging process in cellular level, which play a role in inflammatory and degenerative diseases and abnormal cell growth (30). In such context, foods with anti-inflammatory effects can play a key role in protection against reactive oxygen species and control process of aging, apoptosis, tumor cell growth, and cell injury (31).

The main strength of this study is the large sample size, which helps to generalize the results. This study had several limitations that need to be considered. Since diet is a very complex and potentially modifiable exposure, it is very difficult to elicit information on dietary intake. In addition, human diet is a potentially modifiable exposure; as a result, it is difficult to attribute the etiology of cancer to a single nutrient. Thus, in the present study, dietary patterns were preferred to be assessed. On the other hand, the Food Frequency questionnaire (FFQ) has been widely 
used to capture habitual dietary intake, while its accuracy still remains a concern.

\section{Conclusion}

Our findings suggest that avoiding the Western dietary patterns and diet with inflammatory potential and adherence to the Mediterranean diet pattern may be helpful in reducing the chances of cancer incidence. Further investigations are required to obtain a broader insight into cancer determinants in Iranian populations.

\section{Conflict of Interests}

The authors declare that they have no competing interests.

\section{References}

1. Ferlay J, Soerjomataram I, Dikshit R, Eser S, Mathers C, Rebelo M, et al. Cancer incidence and mortality worldwide: sources, methods and major patterns in GLOBOCAN 2012. Int J Cancer. 2015;136(5):E35986.

2. Giacosa A, Barale R, Bavaresco L, Gatenby P, Gerbi V, Janssens J, et al. Cancer prevention in Europe: the Mediterranean diet as a protective choice. Eur J Cancer Prev. 2013;22(1):90-5.

3. Thun MJ, DeLancey JO, Center MM, Jemal A, Ward EM. The global burden of cancer: priorities for prevention. Carcinogenesis. 2010;31(1):100-10.

4. Feng F, Wei Y, Zheng K, Li Y, Zhang L, Wang T, et al. Comparison of epidemiological features, clinicopathological features, and treatments between premenopausal and postmenopausal female breast cancer patients in western China: a retrospective multicenter study of 15,389 female patients. Cancer Med. 2018.

5. Global, regional, and national comparative risk assessment of 79 behavioural, environmental and occupational, and metabolic risks or clusters of risks, 1990-2015: a systematic analysis for the Global Burden of Disease Study 2015. Lancet (London, England). 2016;388(10053):1659-724.

6. Chlebowski RT, Aragaki AK, Anderson GL, Simon MS, Manson JE, Neuhouser ML, et al. Association of Low-Fat Dietary Pattern With Breast Cancer Overall Survival: A Secondary Analysis of the Women's Health Initiative Randomized Clinical Trial. JAMA Oncol. 2018:e181212.

7. Clutter Snyder D, Sloane R, Haines PS, Miller P, Clipp EC, Morey $\mathrm{MC}$, et al. The Diet Quality Index-Revised: a tool to promote and evaluate dietary change among older cancer survivors enrolled in a home-based intervention trial. J Am Diet Assoc. 2007;107(9):151929.

8. Kim NH, Song S, Jung SY, Lee E, Kim Z, Moon HG, et al. Dietary pattern and health-related quality of life among breast cancer survivors. BMC Women's Health 2018;18(1):65.

9. Park SY, Boushey CJ, Wilkens LR, Haiman CA, Le Marchand L. High-Quality Diets Associate With Reduced Risk of Colorectal Cancer: Analyses of Diet Quality Indexes in the Multiethnic Cohort. Gastroenterology. 2017;153(2):386-94.e2.

10. Jiao L, Chen L, White DL, Tinker L, Chlebowski RT, Van Horn LV, et al. Low-fat Dietary Pattern and Pancreatic Cancer Risk in the Women's Health Initiative Dietary Modification Randomized Controlled Trial. BMC Womens Health. 2018;110(1).

11. Prentice RL, Thomson CA, Caan B, Hubbell FA, Anderson GL, Beresford SA, et al. Low-fat dietary pattern and cancer incidence in the Women's Health Initiative Dietary Modification Randomized Controlled Trial. J Natl Cancer Inst. 2007;99(20):1534-43.

12. Fung TT, Hu FB, Schulze M, Pollak M, Wu T, Fuchs CS, et al. A dietary pattern that is associated with $\mathrm{C}$-peptide and risk of colorectal cancer in women. Cancer Causes Control. 2012;23(6):959-65.

13. Lu S, Qian Y, Huang X, Yu H, Yang J, Han R, et al. The association of dietary pattern and breast cancer in Jiangsu, China: A populationbased case-control study. PLoS One. 2017;12(9):e0184453.

14. Fabiani R, Minelli L, Bertarelli G, Bacci S. A Western Dietary Pattern Increases Prostate Cancer Risk: A Systematic Review and Meta-Analysis. Nutrients. 2016;8(10).

15. Newby PK, Tucker KL. Empirically derived eating patterns using factor or cluster analysis: a review. Nutr Rev. 2004;62(5):177-203.

16. Kant AK. Dietary patterns and health outcomes. J Am Diet Assoc. 2004;104(4):615-35.

17. Turati F, Carioli G, Bravi F. Mediterranean Diet and Breast Cancer Risk. 2018;10(3).

18. Couto E, Boffetta P, Lagiou P, Ferrari P, Buckland G, Overvad K, et al. Mediterranean dietary pattern and cancer risk in the EPIC cohort. Br J Cancer. 2011;104(9):1493-9.

19. Shacter E, Weitzman SA. Chronic inflammation and cancer. Oncology (Williston Park, NY). 2002;16(2):217-26,29; discussion 302.

20. Shivappa N, Steck SE, Hurley TG, Hussey JR, Hebert JR. Designing and developing a literature-derived, population-based dietary inflammatory index. Public Health Nutr,. 2014;17(8):1689-96.

21. Shivappa N, Hebert JR, Steck SE, Hofseth LJ, Shehadah I, BaniHani KE, et al. Dietary Inflammatory Index and odds of colorectal cancer in a case-control study from Jordan. Appl Physiol Nutr Metab. 2017.

22. Shivappa N, Prizment AE, Blair CK, Jacobs DR, Jr., Steck SE, Hebert JR. Dietary inflammatory index and risk of colorectal cancer in the Iowa Women's Health Study. Cancer Epidemiol Biomarkers Prev. 2014;23(11):2383-92.

23. Tabung FK, Steck SE, Ma Y, Liese AD, Zhang J, Caan B, et al. The association between dietary inflammatory index and risk of colorectal cancer among postmenopausal women: results from the Women's Health Initiative. Cancer Causes Control. 2015;26(3):399-408.

24. Wirth MD, Shivappa N, Steck SE, Hurley TG, Hebert JR. The dietary inflammatory index is associated with colorectal cancer in the National Institutes of Health-American Association of Retired Persons Diet and Health Study. Br. J. Nutr.. 2015;113(11):1819-27.

25. Shivappa N, Blair CK, Prizment AE, Jacobs DR, Jr., Hebert JR Dietary inflammatory index and risk of renal cancer in the Iowa Women's Health Study. Eur J Nutr. 2017.

26. Shivappa N, Zucchetto A, Serraino D, Rossi M, La Vecchia C, Hebert JR. Dietary inflammatory index and risk of esophageal squamous cell cancer in a case-control study from Italy. Cancer Causes Control. 2015;26(10):1439-47.

27. Shivappa N, Hebert JR, Rashidkhani B. Dietary Inflammatory Index and Risk of Esophageal Squamous Cell Cancer in a Case-Control Study from Iran. Nutr Cancer. 2015;67(8):1253-9.

28. Shivappa N, Hebert JR, Polesel J, Zucchetto A, Crispo A, Montella $\mathrm{M}$, et al. Inflammatory potential of diet and risk for hepatocellular cancer in a case-control study from Italy. Br. J. Nutr. 2016;115(2):324-31.

29. Hodge AM, Bassett JK, Shivappa N, Hebert JR, English DR, Giles GG, et al. Dietary inflammatory index, Mediterranean diet score, and lung cancer: a prospective study. Cancer Causes Control. 2016;27(7):907-17.

30. Graffouillere L, Deschasaux M, Mariotti F, Neufcourt L, Shivappa $\mathrm{N}$, Hebert JR, et al. The Dietary Inflammatory Index Is Associated with Prostate Cancer Risk in French Middle-Aged Adults in a Prospective Study. J Nutr. 2016.

31. Shivappa N, Jackson MD, Bennett F, Hebert JR. Increased Dietary Inflammatory Index (DII) Is Associated With Increased Risk of Prostate Cancer in Jamaican Men. Nutr Cancer. 2015;67(6):941-8.

32. Shivappa N, Bosetti C, Zucchetto A, Montella M, Serraino D, La Vecchia C, et al. Association between dietary inflammatory index and prostate cancer among Italian men. Br. J. Nutr... 2015;113(2):278-83.

33. Shivappa N, Blair CK, Prizment AE, Jacobs DR, Hebert JR. Prospective study of the dietary inflammatory index and risk of breast cancer in postmenopausal women. Mol Nutr Food Res. 2016.

34. Shivappa N, Hebert JR, Rosato V, Montella M, Serraino D, La Vecchia C. Association between the dietary inflammatory index and breast cancer in a large Italian case-control study. Mol Nutr Food Res. 2017;61(3).

35. Pourshams A, Khademi H, Malekshah AF, Islami F, Nouraei M, Sadjadi AR, et al. Cohort Profile: The Golestan Cohort Study--a prospective study of oesophageal cancer in northern Iran. Int J Epidemiol. 2010;39(1):52-9.

36. Golozar A, Khademi H, Kamangar F, Poutschi H, Islami F, Abnet $\mathrm{CC}$, et al. Diabetes mellitus and its correlates in an Iranian adult population. PloS One. 2011;6(10):e26725.

37. Islami F, Kamangar F, Nasrollahzadeh D, Aghcheli K, Sotoudeh M, Abedi-Ardekani B, et al. Socio-economic status and oesophageal cancer: results from a population-based case-control study in a high- 
risk area. Int. J. Epidemiol.. 2009;38(4):978-88.

38. Malekshah AF, Kimiagar M, Saadatian-Elahi M, Pourshams A, Nouraie M, Goglani G, et al. Validity and reliability of a new food frequency questionnaire compared to $24 \mathrm{~h}$ recalls and biochemical measurements: pilot phase of Golestan cohort study of esophageal cancer. Eur J Clin Nutr. 2006;60(8):971-7.

39. McCann SE, Marshall JR, Brasure JR, Graham S, Freudenheim JL. Analysis of patterns of food intake in nutritional epidemiology: food classification in principal components analysis and the subsequent impact on estimates for endometrial cancer. Public Health Nutr. 2001;4(5):989-97.

40. Gerber M. The comprehensive approach to diet: a critical review. J Nutr. 2001;131(11 Suppl):3051s-5s.

41. Hormozdiari H, Day NE, Aramesh B, Mahboubi E. Dietary factors and esophageal cancer in the Caspian Littoral of Iran. Cancer Res. 1975;35(11 Pt. 2):3493-8.

42. Albuquerque RC, Baltar VT, Marchioni DM. Breast cancer and dietary patterns: a systematic review. Nutr Rev. 2014;72(1):1-17.

43. Feng YL, Shu L, Zheng PF, Zhang XY, Si CJ, Yu XL, et al. Dietary patterns and colorectal cancer risk: a meta-analysis. Eur J Cancer Prev. 2017;26(3):201-11.

44. Grosso G, Bella F, Godos J, Sciacca S, Del Rio D, Ray S, et al. Possible role of diet in cancer: systematic review and multiple metaanalyses of dietary patterns, lifestyle factors, and cancer risk. Nutr Rev. 2017;75(6):405-19.

45. Banikazemi Z, Haji HA, Mohammadi M, Taheripak G, Iranifar E, Poursadeghiyan $\mathrm{M}$, et al. Diet and cancer prevention: Dietary compounds, dietary MicroRNAs, and dietary exosomes. J Cell Biochem. 2018;119(1):185-96.

46. Haraldsdottir A, Torfadottir J, Birgisdottir B, Valdimarsdottir U, Aspelund T, Tryggvadottir L, et al. Dietary pattern in late life and risk of breast cancerAlfheidur Haraldsdottir. Eur J Public Health. 2017;27(suppl-3).

47. Goetz-Perry C. A low fat dietary pattern intervention did not reduce breast cancer, colorectal cancer, or CVD in postmenopausal women. Evid Based Nurs. 2006;9(4):112-3.

48. Abid Z, Cross AJ, Sinha R. Meat, dairy, and cancer. Am J Clin Nutr. 2014;100 Suppl 1:386s-93s.

49. Schwingshackl L, Hoffmann G. Mediterranean dietary pattern, inflammation and endothelial function: a systematic review and metaanalysis of intervention trials. Nutr Metab Cardiovasc Dis. 2014;24(9):929-39.

50. Negri E, La Vecchia C, Franceschi S, D'Avanzo B, Talamini R, Parpinel M, et al. Intake of selected micronutrients and the risk of breast cancer. Int. J. Cancer. 1996;65(2):140-4.

51. Sofi F, Abbate R, Gensini GF, Casini A. Accruing evidence on benefits of adherence to the Mediterranean diet on health: an updated systematic review and meta-analysis. Am J Clin Nutr. 2010;92(5):1189-96.

52. Galland L. Diet and inflammation. Nutr Clin Pract. 2010;25(6):63440.

53. Giugliano D, Ceriello A, Esposito K. The effects of diet on inflammation: emphasis on the metabolic syndrome. J Am Coll Cardiol. 2006;48(4):677-85. 